\title{
Surgical treatment of epiphora with eyelid retraction after a lower eyelid surgery
}

\author{
Jae Hun Lee, MD (D), Nam Yeong Kim, MD (iD, Hee Bae Ahn, MD, PhD (iD \\ Department of Ophthalmology, Dong-A University College of Medicine, Busan, Rep. of Korea
}

Background: Because of the rapidly increasing demand for cosmetic surgery in Korea, the number of patients presenting with epiphora after lower eyelid surgery and diagnosed with retraction is currently increasing.

Objective: To evaluate the effect of the lateral tarsal strip procedure (LTS) in patients with epiphora and eyelid retraction after lower eyelid surgery.

Methods: A total of 145 eyes of 81 patients who reported to our hospital between January 2010 and February 2020 were included in the study. Twenty-six eyes of 17 patients underwent LTS, and 119 eyes of 64 patients underwent LTS combined with silicone tube intubation or endonasal dacryocystorhinostomy (DCR). The pre- and postoperative symptoms, margin reflex distance 2 (MRD2), tear meniscus height (TMH), and complications were retrospectively compared.

Results: Seventy-two of the 81 patients (88.9\%) showed improvements in epiphora. The improvements in TMH in the combined surgery and single surgery groups were $0.45 \pm 0.28$ and $0.44 \pm 0.18 \mathrm{~mm}$, respectively, showing postoperative improvements in both groups. MRD2 also improved to $0.62 \pm 0.66$ and $0.27 \pm 0.68 \mathrm{~mm}$ in the combined surgery and single surgery groups, respectively. No significant difference was observed between the two groups.

Conclusion: LTS is an effective surgical method for epiphora in patients experiencing eyelid retraction after lower eyelid surgery. Its combination with silicone tube intubation or endonasal DCR is considered effective for lacrimal pathway disorders.

Keywords: endonasal dacryocystorhinostomy; epiphora; eyelid retraction; lateral tarsal strip; silicone tube intubation

\section{Introduction}

For a patient with epiphora, it is necessary to first determine whether the cause is the overproduction or the impaired drainage of tears. The causes of overproduction include emotions, central nervous system problems, irritation or pain of the cornea or conjunctiva (as with dry eye), irritation of the lacrimal gland nerve, inflammation of the lacrimal gland, and tumors. Tests, such as lacrimal syringing and lacrimal scintigraphy, may be performed to assess whether the lacrimal drainage pathway, which includes the punctum, lacrimal canaliculus, lacrimal sac, and nasolacrimal duct, is obstructed. If the lacrimal drainage pathway is patent, dysfunction in the tear pump should be suspected [1,2]. An incomplete closure of the eyelid caused by trauma, scar, facial palsy, scleroderma, or burn may decrease the function of the lacrimal pump. Moreover, for cases of eyelid retraction, tears may be discharged because of the movement of the punctum $[3,4]$.

Ectropion refers to eyelid eversion, and it most often affects the lower eyelid. Ectropion has several etiologies, which may be degenerative, related to scarring, paralytic, and congenital. Because of the rapidly increasing demand for cosmetic surgery in

Received September 7, 2020; Revised November 15, 2020; Accepted December 4, 2020

Corresponding author: Hee Bae Ahn

E-mail: hbahn@dau.ac.kr

This is an Open Access article distributed under the terms of the Creative Commons Attribution Non-Commercial License (http://creativecommons.org/licenses/by-nc/4.0), which permits unrestricted non-commercial use, distribution, and reproduction in any medium, provided the original work is properly cited.

Copyright ( 2020 Korean Society of Korean Cosmetic Surgery and Medicine (KSKCS \& KCCS). 
Korea, the patients presenting with epiphora after lower eyelid surgery and those diagnosed with ectropion are increasing [5]. Surgical methods for the correction of ectropion and eyelid retraction include the lateral tarsal strip (LTS) procedure, medial spindle operation, medial or lateral canthopexy, and lazy-T procedure. Among these, LTS is the easiest to perform and the most effective. LTS strengthens the eyelids horizontally and improves lacrimal pump function [6-8]. This study assessed the efficacies of LTS alone and LTS combined with silicone tube intubation or endonasal dacryocystorhinostomy (DCR) in patients with eyelid retraction and a history of lower eyelid surgery.

\section{Materials and methods}

A total of 88 patients (24 male, 64 female) with a history of lower eyelid surgery who visited the hospital for epiphora between January 2010 and February 2020 were retrospectively evaluated. Among these, 7 patients with eye diseases, such as eyelid retraction, dacryocystitis, conjunctivitis, conjunctival stones, dry eyes, or a history of lower eyelid surgery due to trauma, were excluded from the study [9]. Thus, a total of 81 patients (19 male, 62 female) were included in this study. This study was approved by the Institutional Review Board of the Dong-A University Hospital (IRB No. DAUHIRB-20-147). Written informed consent was obtained.

The margin reflex distance 2 (MRD2) and tear meniscus height $(\mathrm{TMH})$ of all patients were evaluated, and a lacrimal syringing test was performed. Pre- and postoperative TMH were measured by a single observer using a slit-lamp microscope. The distance from the lower eyelid to the edge of the tear meniscus along the ocular surface was measured with a $0.2-\mathrm{mm}$ or $1.0-\mathrm{mm}$ slit beam. With a TMH of $\leq 0.5 \mathrm{~mm}$, a $0.2-\mathrm{mm}$ slit beam was used for measurement [10]. The Image J program (NIH, Bethesda, MD, USA) was used to accurately evaluate MRD2 (Fig. 1).

All eyelid retraction procedures were performed by a single surgeon. If lacrimal pathway pathology, such as punctal stric- ture, canalicular stenosis or obstruction, or nasolacrimal duct obstruction, was detected by the lacrimal syringing test, silicone tube intubation surgery or endonasal DCR surgery was performed together with LTS. The single surgery group comprised patients who underwent LTS alone. Furthermore, the patients who underwent LTS in combination with silicone tube intubation or endonasal DCR surgery were classified as the combined surgery group.

For LTS, cantholysis or canthus sparing was selected by the surgeon depending on the degree of eyelid relaxation. Cantholysis was performed as described by Jordan and Anderson [8]. After lateral canthotomy was performed, the inferior crus of the lateral canthal tendon was cut to separate the lower eyelid from the inner and lateral edges. After separating the anterior and posterior lamellae, the lateral tarsal conjunctiva was cut to make a tarsal strip. The lateral lower lid was pulled laterally afterward, and the excess amount of tarsal strip was measured and excised. Finally, the tarsal strip was reattached deep into the periosteum with 5-0 Dacron (Dacron; E. I. du Pont de Nemours \& Co. Inc., Wilmington, Delaware, USA).

Canthus sparing was performed as described by Lemke et al. [11]. An incision was made 6-8 $\mathrm{mm}$ away from the lateral canthus. The orbicularis oculi muscle was peeled off, and a periosteal flap was made at the lateral corner of the orbital rim. Subsequently, the periosteal flap was raised to $2-3 \mathrm{~mm}$ of the lateral orbital wall near the lateral orbital tubercle of Whitnall using a periosteal elevator. The periosteal flap was attached to the canthal ligament.

The procedure for silicone tube intubation began with nasal packing with Bosmin gauze (JEIL PHARMACEUTICAL CO., LTD., Seoul, Korea) in the inferior meatus of the nose. After the punctum was dilated gently, the lacrimal probe was passed through the lacrimal canaliculus. A silicone tube was inserted into the lower punctum, and the tip was extracted with Alligator forceps through the nasal speculum. Upper intubation was performed using the same method. The upper and lower silicone tubes were knotted and tied, using 6-0 nylon in the left nose,
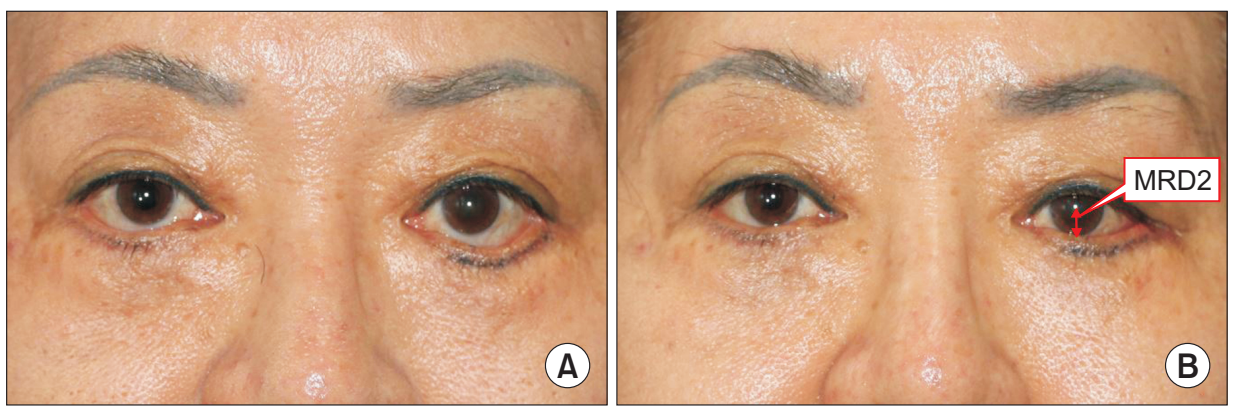

Fig. 1. Preoperative (A) and postoperative (B) photographs of a patient with lateral tarsal strip surgery. Preoperative and postoperative MRD2 are measured using Image J program. MRD2, margin reflex distance 2 . 
Table 1. Preoperative and postoperative tear meniscus heights

\begin{tabular}{lccc}
\hline $\begin{array}{c}\text { Tear meniscus } \\
\text { height }\end{array}$ & $\begin{array}{c}\text { Combined surgery } \\
\text { group }(\mathbf{n}=119)\end{array}$ & $\begin{array}{c}\text { Single surgery } \\
\text { group }(\mathbf{n = 2 6})\end{array}$ & $\begin{array}{c}\text { Total } \\
(\mathbf{n}=\mathbf{1 4 5})\end{array}$ \\
\hline Preoperative $(\mathrm{mm})$ & $0.57 \pm 0.27$ & $0.61 \pm 0.15$ & $0.58 \pm 0.25$ \\
Postoperative $(\mathrm{mm})$ & $0.12 \pm 0.14$ & $0.17 \pm 0.13$ & $0.13 \pm 0.14$ \\
p-value & $<0.001^{*}$ & $<0.001^{*}$ & $<0.001^{*}$
\end{tabular}

Values are presented as mean \pm SD.

*Wilcoxon signed rank test.

and attached to the lateral wall of the nasal antrum.

Endonasal DCR also began with nasal packing, punctal dilation, and probing. The thinnest area of the inner mucosa was found using laser transillumination. The lacrimal bone was removed, using drilling and a diode laser, through the canaliculi. Endoscopy-guided silicone tube intubation was performed using the same method as that used for silicone tube intubation.

The silicone tube inserted during silicone tube intubation or endonasal DCR was removed 3-6 months after the surgery, depending on the presence of symptoms.

The surgical results were based on epiphora symptoms and changes in TMH and MRD2 1-month post-operation and, during re-operation, as well as the complications.

\section{Statistical analysis}

The collected data were analyzed using IBM SPSS ver. 23.0 software (IBM Corp., Armonk, NY, USA). Descriptive statistics were used to evaluate the frequencies, percentages, means, and standard deviations of sex, age, follow-up duration (months), and the type of eye treatment (monocular or binocular). The differences in $\mathrm{TMH}$ and MRD2 pre- and post-operation were analyzed using the Wilcoxon signed-rank test. The pre- and postoperative TMH and MRD2 for the two surgery groups were compared using the Mann-Whitney U test. p-value of less than 0.05 were considered significant.

\section{Results}

Among the 145 eyes of 81 patients, 26 eyes of 17 patients underwent LTS, and 119 eyes of 64 patients underwent LTS combined with silicone tube intubation or endonasal DCR.

The mean age of the patients was $65.41 \pm 8.91$ years, and the mean follow-up duration was $6.29 \pm 3.29$ months. Nineteen male (23.5\%) and 62 female (76.5\%) were included in the study. Eighteen subjects $(22.2 \%)$ underwent monocular treatment, and $63(77.8 \%)$ underwent binocular treatment. Overall, 119 eyes $(82.1 \%)$ were included in the combined surgery group, whereas 26 eyes (17.9\%) were included in the single surgery group.
Table 2. Preoperative and postoperative margin reflex distance 2 (MRD2)

\begin{tabular}{lccc}
\hline \multicolumn{1}{c}{ MRD2 } & $\begin{array}{c}\text { Combined surgery } \\
\text { group }(\mathbf{n}=119)\end{array}$ & $\begin{array}{c}\text { Single surgery } \\
\text { group }(\mathbf{n}=\mathbf{2 6})\end{array}$ & $\begin{array}{c}\text { Total } \\
(\mathbf{n}=\mathbf{1 4 5})\end{array}$ \\
\hline Preoperative (mm) & $5.38 \pm 0.82$ & $5.90 \pm 0.89$ & $5.47 \pm 0.86$ \\
Postoperative $(\mathrm{mm})$ & $4.76 \pm 0.61$ & $5.64 \pm 1.05$ & $4.91 \pm 0.78$ \\
p-value & $<0.001^{*}$ & $0.031^{*}$ & $<0.001^{*}$ \\
\hline
\end{tabular}

Values are presented as mean \pm SD.

*Wilcoxon signed rank test.

Successful results were observed in 72 of 81 patients (88.9\%). In the combined surgery group, TMH and MRD2 improved by $0.45 \pm 0.28 \mathrm{~mm}(\mathrm{p}<0.001)$ and $0.62 \pm 0.66 \mathrm{~mm}(\mathrm{p}<0.001)$, respectively, both changes were statistically significant. TMH and MRD2 improved by $0.44 \pm 0.18 \mathrm{~mm}(\mathrm{p}<0.001)$ and $0.27 \pm 0.68 \mathrm{~mm}$ $(\mathrm{p}=0.031)$, respectively, in the single surgery group; these were also statistically significant.

TMH measured at the last visit after surgery was statistically significant $(\mathrm{p}=0.009)$ at $0.12 \pm 0.14 \mathrm{~mm}$ and $0.17 \pm 0.13 \mathrm{~mm}$ in the combined surgery and single surgery groups, respectively. MRD2 measured was $4.76 \pm 0.61 \mathrm{~mm}$ in the combined surgery group and $5.64 \pm 1.05 \mathrm{~mm}$ in the single surgery group; these values were also statistically significant $(\mathrm{p}<0.001)$.

The TMH improvements in the combined surgery group and single surgery group were $0.45 \pm 0.28 \mathrm{~mm}$ and $0.44 \pm 0.18 \mathrm{~mm}$, respectively. The difference between the TMH improvements in the two groups was not statistically significant $(\mathrm{p}=0.780)$. MRD2 was $0.62 \pm 0.66 \mathrm{~mm}$ and $0.27 \pm 0.68 \mathrm{~mm}$ in the combined surgery and single surgery groups, respectively. The difference between the MRD2 values of the two groups was not statistically significant (Table 1, 2).

In 9 of 81 patients, anatomical correction was achieved. However, epiphora persisted because of dryness, and, treatments, such as artificial tears, were administered.

\section{Discussion}

Epiphora is caused by the overproduction or impaired drainage of tears. There are several hypotheses on the mechanism of the lacrimal pump underlying the release of tears. The first is Rosengren-Doane's active palpebral canalicular pump theory, which states that the lacrimal ampulla pushes tears into the lacrimal sac while the eye is blinking. Subsequently, the negative pressure inside the lacrimal sac pulls the tear. Another widely accepted theory, the tear pump theory proposed by Jones, states that the actions of muscles, such as the preseptal orbicularis oculi and the pretarsal orbicularis of the eyelid, move tears 
to the lacrimal drainage system located above and below [1215].

According to the reports by Jones and Linn [14], epiphora was caused by disorders of the tear pump in $11.1 \%$ of patients. Among them, the most common cause was senile relaxation of the lower eyelid (5.6\%). Furthermore, Liu and Stasior [16] measured lower eyelid laxity and reported its association with symptoms such as foreign body sensation, chronic tearing, and chronic recurrent conjunctivitis. Keratitis and other inflammatory conditions may lead to excessive tear discharge and mild punctal ectropion or stenosis accompanied by lower eyelid laxity. Thus, finding and correcting the exact cause of epiphora is necessary.

Several surgical methods have been developed and described in the literature for the correction of lower eyelid laxity $[2,7,11,17$. Vick et al. [2] performed LTS on 34 eyes of 21 patients with functional epiphora and reported that only 3 patients did not show improvement of symptoms. LTS improves epiphora by enhancing tear pump function by decreasing lower eyelid laxity [18]. When the lower eyelid is strengthened, the orbicularis oculi muscle acts more effectively when closing the eyes, and this sends tears to the punctum. It also increases the pulling force on the lacrimal sac wall, which increases the negative pressure in the lacrimal sac [19].

Another study by Chung and Park [20] treated 12 eyes of 9 patients who had functional obstruction of the lacrimal drainage system with conjunctivodacryocystorhinostomy and reported satisfactory results for all of them. In a study by Wormald and Tsirbas [21], 27 out of 32 patients (84.4\%) with functional obstruction of the lacrimal drainage system who underwent DCR showed improvement. As such, it has been recently recommended that LTS should be performed alone or in combination with other surgeries in patients with functional epiphora due to lower eyelid laxity [8].

In the current study, the surgical effects of the single and combined surgery were compared. Pre- and postoperative TMH showed significant improvement in all patients in both single and combined surgery groups. MRD2 also significantly improved in all patients in both groups. However, after comparing both groups, statistically insignificant results were obtained in the combined surgery group. This may be attributed to the differences in sample size, as the combined surgery group had more patients. Therefore, a larger sample size is recommended in future studies. It is also thought that the silicone tubes expanded the punctum, leading to better results in the combined surgery group [10].
When performing lower eyelid surgery for cosmetic or other purposes, complications such as epiphora and eyelid retraction should be considered. In our study, $79.0 \%$ of patients who underwent LTS surgery after lower lid surgery underwent combined silicone tube intubation or endonasal DCR. Low eyelid surgery is performed frequently in older adults, and lacrimal pathway problems should be ruled out. Therefore, lacrimal syringing tests should be performed before lower lid surgery.

\section{Conflicts of interest}

The authors have nothing to disclose.

\section{References}

1. Rosenstock T, Hurwitz JJ. Functional obstruction of the lacrimal drainage passages. Can J Ophthalmol 1982;17:249-55.

2. Vick VL, Holds JB, Hartstein ME, Massry GG. Tarsal strip procedure for the correction of tearing. Ophthalmic Plast Reconstr Surg 2004;20:37-9.

3. Jones LT. Epiphora: its causes and new surgical procedures for its cure; a preliminary report. Am J Ophthalmol 1954;38:82431 .

4. Jones LT. An anatomical approach to problems of the eyelids and lacrimal apparatus. Arch Ophthalmol 1961;66:111-24.

5. Vallabhanath P, Carter SR. Ectropion and entropion. Curr Opin Ophthalmol 2000;11:345-51.

6. Anderson RL. Tarsal strip procedure for correction of eyelid laxity and canthal malposition in the anophthalmic socket. Ophthalmology 1981;88:895-903.

7. Anderson RL, Gordy DD. The tarsal strip procedure. Arch Ophthalmol 1979;97:2192-6.

8. Jordan DR, Anderson RL. The lateral tarsal strip revisited. The enhanced tarsal strip. Arch Ophthalmol 1989;107:604-6.

9. Nowinski TS, Anderson RL. The medial spindle procedure for involutional medial ectropion. Arch Ophthalmol 1985;103:1750-3.

10. Ryu WY, Suh JY, Ahn HB. Lateral tarsal strip procedure and silicone tube intubation on the functional nasolacrimal duct obstruction. J Korean Ophthalmol Soc 2010;51:1174-8.

11. Lemke BN, Cook BE Jr, Lucarelli MJ. Canthus-sparing ectropion repair. Ophthalmic Plast Reconstr Surg 2001;17:161-8.

12. Doane MG. Blinking and the mechanics of the lacrimal drainage system. Ophthalmology 1981;88:844-51.

13. Jones LT. Epiphora. II. Its relation to the anatomic structures and surgery of the medial canthal region. Am J Ophthalmol 
1957;43:203-12.

14. Jones LT, Linn ML. The diagnosis of the causes of epiphora. Am J Ophthalmol 1969;67:751-4.

15. Hill JC. Treatment of epiphora owing to flaccid eyelids. Arch Ophthalmol 1979;97:323-4.

16. Liu D, Stasior OG. Lower eyelid laxity and ocular symptoms. Am J Ophthalmol 1983;95:545-51.

17. Bick MW. Surgical management of orbital tarsal disparity. Arch Ophthalmol 1966;75:386-9.

18. Cannon PS, Sadiq SA. Can eyelid taping predict the benefit of a lateral tarsal strip procedure in patients with eyelid laxity and functional epiphora? Ophthalmic Plast Reconstr Surg 2009;25:194-6.

19. Becker BB. Tricompartment model of the lacrimal pump mechanism. Ophthalmology 1992;99:1139-45.

20. Chung WS, Park NG. Functional obstruction of the lacrimal draings system. J Korean Ophthalmol Soc 1995;36:1435-8.

21. Wormald PJ, Tsirbas A. Investigation and endoscopic treatment for functional and anatomical obstruction of the nasolacrimal duct system. Clin Otolaryngol Allied Sci 2004;29:3526. 